\title{
Role of Nuclear Factor Erythroid 2-Related Factor 2 in Chronic Obstructive Pulmonary Disease
}

Woo Ho Ban, M.D. ${ }^{1}$ (D) and Chin Kook Rhee, M.D., Ph.D. ${ }^{2}$ (DD

${ }^{1}$ Division of Pulmonary and Critical Care and Sleep Medicine, Department of Internal Medicine, Eunpyeong St. Mary's Hospital, College of Medicine, The Catholic University of Korea, Seoul, ${ }^{2}$ Division of Pulmonary and Critical Care Medicine, Department of Internal Medicine, Seoul St. Mary's Hospital, College of Medicine, The Catholic University of Korea, Seoul, Republic of Korea

\section{Abstract}

Chronic obstructive pulmonary disease (COPD) is characterized by airflow limitation due to chronic airway inflammation and destruction of the alveolar structure from persistent exposure to oxidative stress. The body has various antioxidant mechanisms for efficiently coping with such oxidative stress. The nuclear factor erythroid 2-related factor 2 (Nrf2)-antioxidant response element (ARE) is a representative system. Dysregulation of the Nrf2-ARE pathway is responsible for the development and promotion of COPD. Furthermore, COPD severity is also closely related to this pathway. There has been a clinical impetus to use Nrf2 for diagnostic and therapeutic purposes. Therefore, in this work, we systematically reviewed the clinical significance of Nrf2 in COPD patients, and discuss the value of Nrf2 as a potential COPD biomarker.

Keywords Chronic Obstructive Pulmonary Disease; Nuclear Factor Erythroid 2-Related Factor 2; Oxidative Stress; Inflammation; Biomarker

\section{Introduction}

Chronic obstructive pulmonary disease (COPD) is associated with persistent respiratory symptoms and airflow limitations due to damage to the airways and alveolar structures caused by exposure to noxious particles or gases ${ }^{1}$. The prevalence, morbidity, and mortality from COPD are very high; thus, COPD represents an important socioeconomic and public health burden ${ }^{2}$. Patients with COPD have several comorbidities, such as coronary artery disease, diabetes, osteoporosis, sarcopenia, and depression, and the ailment is recognized as a systemic disease due to systemic inflammation ${ }^{3,4}$.

Various mechanisms explain the causes of systemic inflammation, the most common of which is oxidative stress $^{5}$. Reactive oxygen species (ROS) form through in vivo metabolic processes that use oxygen as a fuel, as well as during exposure to external or toxic environ- https://doi.org/10.4046/trd.2021.0162 ISSN: $1738-3536$ (Print)/ 2005-6184(Online) Tuberc Respir Dis 2022;85:221-226
Check for
updates

Copyright (C) 2022 The Korean Academy of Tuberculosis and Respiratory Diseases

Address for correspondence Chin Kook Rhee, M.D., Ph.D.

Division of Pulmonary and Critical Care Medicine, Department of Internal Medicine, Seoul St. Mary's Hospital, College of Medicine, The Catholic University of Korea, 222 Banpodaero, Seocho-gu, Seoul 06591, Republic of Korea Phone 82-2-2258-6067 Fax 82-2-599-3589 E-mail chinkook77@gmail.com Received Nov. 3, 2021 Revised Jan. 22, 2022 Accepted Mar. 1, 2022 Published online Mar. 8, 2022

\section{(c) (i) \$ \\ () It is identical to the Creative Commons Attribution Non- Commercial License (http:// creativecommons.org/licenses/ by-nc/4.0/).}

ments $^{6}$. An optimal level of ROS is beneficial for maintaining biological activities by inducing inflammation, immune function, autophagy, and a proper stress response $^{7}$. However, over-activation of ROS leads to DNA damage and cell apoptosis and causes ischemic injury at the tissue level ${ }^{8}$.

Smoking is the main cause of COPD, as it generates excessive oxidative stress, causing alveolar epithelial cell damage and triggering the development and progression of COPD ${ }^{9}$. Several systems in the body fight this excessive oxidative stress. The most representative is the nuclear factor erythroid 2-related factor 2 (Nrf2) pathway, which belongs to the cap ' $n$ ' collar subfamily of basic region leucine zipper transcription factors. Nrf2 induces several drug-metabolizing enzymes, such as glutathione S-transferase and NAD(P) $\mathrm{H}$ :quinone oxidoreductase 1 by upregulating a common DNA sequence called the antioxidant response 
element (ARE) ${ }^{10}$. Nrf2 is degraded proteasomally by its cytosolic inhibitor Kelch-like ECH-associated protein 1 (KEAP1) under basal conditions and is released from KEAP1 under oxidative stress conditions to activate the ARE (Figure 1) ${ }^{11}$.

Nrf2 is a representative antioxidant molecule that has been emphasized for its relevance to COPD and significant association with oxidative stress. Furthermore, research has been actively conducted on the possibility that Nrf2 is a COPD biomarker and on its role as a therapeutic target. In this paper, we review the significance of oxidative stress and Nrf2 in the development and progression of COPD and summarize the value of Nrf2 as a COPD biomarker.

\section{Nrf2 in the Development of COPD}

Long-term exposure of a normal airway to smoking has a fundamental impact on the development of COPD, leading to a chronic inflammatory reaction, resulting from deposition of various inflammatory cells. However, destruction of lung structure and resulting emphysema or small airway obstruction does not occur in all smokers $^{12,13}$. Many hypotheses have been proposed about the causes of COPD in some smokers, including mucus hyper-production due to abnormal immune reactions, small airway obstruction due to excessive tissue repair and airway remodeling; insufficient tissue repair leads

Figure 1. The response of Nrf-2 to oxidative stress. Nrf2: nuclear factor erythroid 2-related factor 2; KEAP1: Kelch-like ECH-associated protein 1; ARE: antioxidant response element.

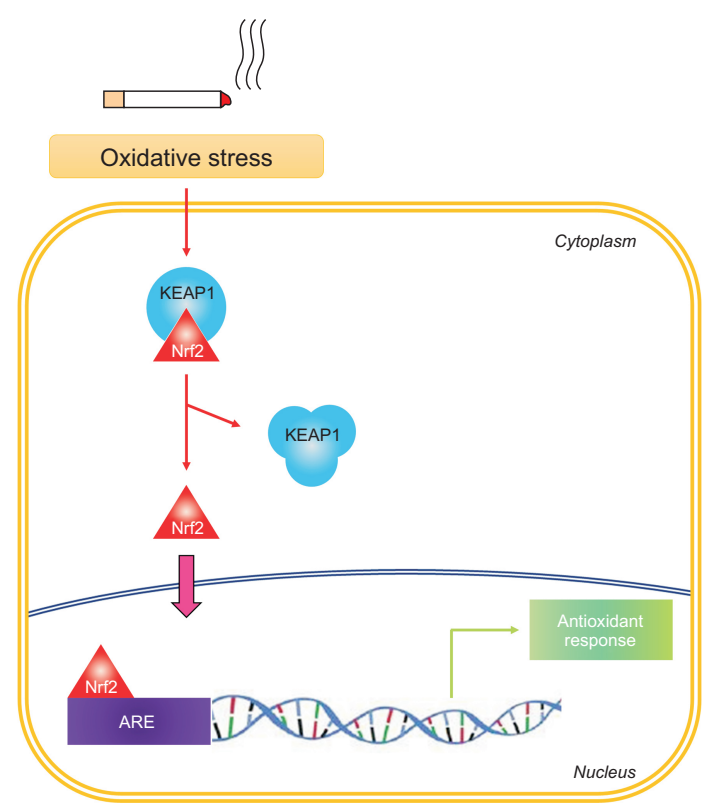

to emphysematous changes ${ }^{14}$.

Especially, many animal studies have been conducted on the role of Nrf2 in the development of emphysema, a major phenotype of COPD. A large number of researchers insist that inadequate tissue repair due to loss of the protective role of Nrf2 leads to emphysema-dominant COPD. Nrf2-knockout mice is a useful tool to evaluate the role of Nrf2. Nrf2-knockout mice with a BALB/c background can be established by specific deletion of the Nrf2 gene segment. Nrf2-knockout mice grow normally and are fertile, but are susceptible to oxidative stress. Nrf2-knockout COPD model was made by intratracheal instillation of elastase or exposure to cigarette smoke (CS) in Nrf2-knockout mice. lizuka et al. ${ }^{15}$ and Ishii et al. ${ }^{16}$ showed that neutrophilic inflammation and elastase activity increase in bronchoalveloar lavage (BAL) fluid after exposing Nrf2-knockout mice to CS, which reduces antioxidant and anti-protease activities. These results suggest that functional loss of Nrf2 is involved in COPD development in smokers $^{15,16}$. In another study conducted by Sussan et al. ${ }^{17}$, destruction of alveolar structure and pulmonary hypertension are attenuated in wild-type Nrf2 mice exposed to CS after oral administration of the triterpenoid CDDO-imidazolide Nrf2 activator.

The role of Nrf2 in the development of COPD has also been studied in humans. Goven et al. ${ }^{18}$ compared the expression of Nrf2 and Keap1 in lung tissues and alveolar macrophages of patients with and without emphysema. They showed that Nrf2 protein expression decreased, whereas the expression of Keap1 increased. They suggested that this altered equilibrium of Nrf2Keap1 and reduction in the expression of sequential antioxidant proteins was the main mechanism of emphysematous changes in smokers ${ }^{18}$. Suzuki et al. ${ }^{19}$ compared Nrf2 expression in alveolar macrophages obtained from BAL fluid among young subjects, older current smokers, and patients with COPD. They found that Nrf2 expression decreased in the macrophages of older current smokers and patients with COPD ${ }^{19}$.

Most of these studies suggest that a lack of tissue repair and chronic inflammation via downregulation of Nrf2 lead to obstruction of small airways and destruction of lung parenchyma, resulting in the development of COPD.

\section{Nrf2 in the Progression of COPD}

The most important factor leading to disease progression during the natural course of COPD is an exacerbation. Frequent exacerbations of COPD affect not only acute symptoms but also reduce long-term lung 
function $^{20}$. Furthermore, an exacerbation increases the morbidity and mortality from COPD ${ }^{21}$. COPD exacerbations are triggered mainly by acute airway inflammation caused by bacterial or viral infection ${ }^{22}$. Most patients with COPD are susceptible to bacterial or viral infections. Studies have shown that oxidative stress resulting from chronic exposure to smoke or noxious particles leads to dysfunction of the alveolar macrophages, which impairs phagocytic activity ${ }^{23}$. Therefore, downregulation of Nrf2 in patients with COPD may lead to excessive oxidative stress, dysfunction of alveolar macrophages, COPD exacerbation, and disease progression. Several studies have experimentally demonstrated this hypothesis. Harvey et al. ${ }^{24}$ showed that pulmonary bacterial clearance of alveolar macrophages by phagocytosis in Nrf2 wild-type and Nrf2-deficient mice improves by activating Nrf2 with the isothiocyanate sulforaphane, suggesting that Nrf2 could be a major target of exacerbation in relation to bacterial clearance. Degradation of the host defense due to reduced Nrf2 has also been studied in viral infections. Cho et al. ${ }^{25}$ showed that clearance of respiratory syncytial virus (RSV) decreases and excessive virus-induced inflammation is induced in Nrf2-deficient mice. Recurrent RSV infection in the COPD mouse model induces inflammatory cytokine production, such as interleukin (IL)-1 $\alpha$, IL-17, and interferon- $\gamma$, as well as the expression of proteases, such as matrix metalloproteinase-2 and -8 , resulting in destruction of lung tissues ${ }^{26}$.

Thus, these studies suggest that a decrease in Nrf2 activity may be a major factor in the progression of COPD (Figure 2). They also suggest that activation of Nrf2 expression may inhibit the progression of COPD. Based on these concepts, Wise et al. ${ }^{27}$ conducted a randomized, double-blind, placebo-controlled trial to determine whether oxidative stress, airway inflammation, and pulmonary function improve with the use of the Nrf2 inducer oral sulforaphane in 89 patients with COPD. However, the effects of sulforaphane in patients with COPD were not demonstrated. It was also unclear whether activation of Nrf2 was ineffective for COPD patients because sulforaphane failed to stimulate the expression of Nrf2 target genes or some other problem. Further clinical trials of COPD using another Nrf2 activator are needed. To date, there has been significant research on the role of Nrf2 in inhibiting COPD progression in vivo, but negative data have been reported for clinical implications. Therefore, further studies are needed to clarify the role of Nrf2 in COPD progression.

\section{Nrf2 and the Severity of COPD}

As stated in the Global Initiative for Chronic Obstructive Lung Disease (GOLD) guidelines, patients with COPD are treated according to disease severity and classified as to their airflow limitation, risk of exacerbation, and symptom burden. However, since the 2017 update, the degree of airflow limitation represented by forced expiratory volume in 1 second $\left(F E V_{1}\right)$ in the GOLD guidelines has been excluded from the patient classification criteria. However, FEV 1 remains a good indicator of an individual patient's clinical outcome ${ }^{28}$. Many studies have classified severity of COPD according to the extent of these airflow limitations, and have investigated whether differences in oxidative stress, proteostasis imbalance, and structural changes in the lung are among them. Min et al. ${ }^{29}$ showed that Nrf2 and histone deacetylase 2 (HDAC2) expression decrease with increasing severity of COPD, according to airflow limitation (GOLD I-IV) in paraffin-embedded lung sections of patients with COPD. They also documented increased oxidative stress and corticosteroid resistance along with COPD severity.

Exacerbation of COPD has been defined as worsening of the respiratory symptom beyond normal day-today variations. Many studies have revealed that COPD exacerbation is closely related to excessive inflammation in response to increased oxidative stress. It is thus important to understand the mechanism of exacerbation and role of Nrf2 during the acute exacerbation. Frequent exacerbations are closely related to the severity of COPD, and long-term macrolide treatment, such as azithromycin, lowers the incidence of exacerbations. Therefore, in the GOLD 2019 guidelines, azithromycin is recommended for use with maximal inhaler treatment for frequent exacerbators ${ }^{30}$. The phosphoinositide 3-kinase (PI3K)/Akt pathway is upregulated by excessive oxidative stress in patients with COPD, which reduces the expression of HDAC2. The use of a macrolide inhibits inflammation by inhibiting the PI3K/ Akt pathway and restoring corticosteroid sensitivity by upregulating $\mathrm{HDAC}^{31,32}$. Nrf2 acts as a key factor downstream of PI3K/Akt. However, the relationship between the PI3K/Akt-Nrf2 pathway and airway and systemic inflammation has not yet been investigated well in severe COPD patients. Therefore, further studies are required to detail the mechanisms of the Nrf2 and $\mathrm{PI} 3 \mathrm{~K} / \mathrm{Akt}$ pathways in patients with severe COPD. 
Figure 2. Nrf2 dysfunction in COPD. (A) In healthy smoker, the activated Nrf2 prevents excessive airway inflammation and apoptosis caused by oxidative stress. (B) In patients with COPD, dysfunctional Nrf2 results in excessive airway inflammation and apoptosis, and causes frequent exacerbation and steroid resistance. Nrf2: nuclear factor erythroid 2-related factor 2; COPD: chronic obstructive pulmonary disease; HDAC: histone deacetylase 2; PI3K: phosphoinositide 3-kinase.
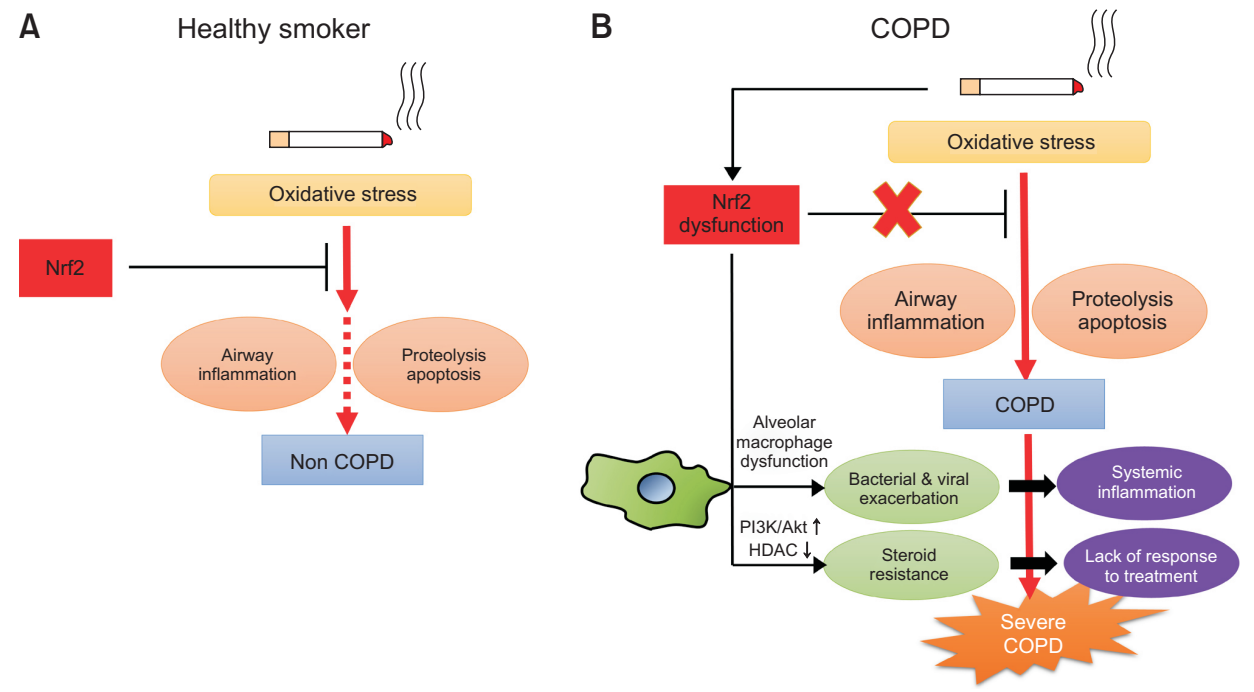

\section{Nrf2 as a Diagnostic and Treatment Target in Patients with COPD}

Some studies have predicted the clinical outcomes, disease severity, and exacerbation risk in patients with COPD using Nrf2, and some have assessed the possible relationships between polymorphisms in the Nrf2 gene and COPD. Yamamoto et al. ${ }^{33}$ compared the polymorphisms of the Nrf2 promoter region in 87 patients with COPD and 50 healthy controls, but they did not find any statistical difference among them. However, in a study of 915 subjects on the general population of Japan, a specific single nucleotide polymorphism of the Nrf2 gene (rs6726395) was associated with a decline in $\mathrm{FEV}_{1}$, particularly in the major allele of smokers ${ }^{34}$. The researchers insisted that smoking affects the Nrf2 polymorphism, which leads to a decrease in $\mathrm{FEV}_{1}$ and ultimately to the development of COPD.

Several studies have reported increased oxidative stress due to impaired Nrf2 expression in the bronchial epithelium and lung tissue of patients with COPD. Yamada et al. ${ }^{35}$ reported that Nrf2 mRNA and protein expression significantly decrease in bronchial epithelial cells of COPD patients. As previously mentioned, Nrf2 expression decreases according to the degree of airway limitation in the lung tissues of patients with $\mathrm{COPD}^{29}$. The attenuation of Nrf2 at the airway and tissue levels causes the development and progression of COPD.
The concept of COPD as a systemic inflammatory disease of oxidative stress, rather than a simple airway inflammatory disease, has implications for peripheral blood Nrf2 levels. Fratta Pasini et al. ${ }^{36}$ showed that Nrf2 expression increases in peripheral blood mononuclear cells of patients with COPD, suggesting that this is a systemic response to oxidative stress that persists even after the cessation of smoking. Indeed, plasma Nrf2 levels are negatively correlated with $\mathrm{FEV}_{1}$ and $\mathrm{FEV}_{1} /$ forced vital capacity but increase during exacerbation in COPD patients ${ }^{37}$. The findings of these two studies contrast with the general trend in Nrf2 expression described above. However, the Nrf2 expression pattern may differ between the airway and systemic inflammatory process in COPD patients, so further study is needed to resolve the precise mechanism.

\section{Conclusion}

The key molecule controlling oxidative stress from smoking or noxious particles is Nrf2. Genetic changes and dysfunction of Nrf2 induce chronic inflammation, apoptosis, and proteolysis of alveolar cells, resulting in changes in the airway and lungs. Furthermore, Nrf2 accelerates disease severity and the progression of COPD (Figure 3). Nrf2 has been emphasized as a potential biomarker for predicting the initiation, severity, and degree of airway or systemic inflammation in COPD, even as a treatment target to improve airway limitations or 
Figure 3. Role of Nrf2 in the development and progression of COPD. Nrf2: nuclear factor erythroid 2-related factor 2; COPD: chronic obstructive pulmonary disease.

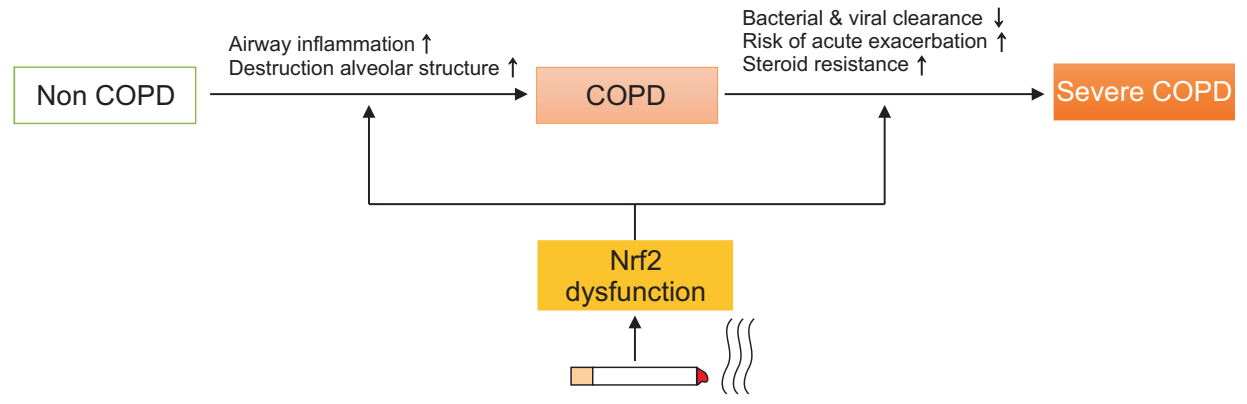

prevent deterioration of COPD. However, COPD is not a simple airway disease but rather a systemic disease, and Nrf2 is more complex when responding to local and systemic oxidative stress. Therefore, additional studies to uncover the precise mechanisms of Nrf2 are needed to use Nrf2 as a diagnostic and therapeutic biomarker for COPD in clinical practice.

\section{Authors' Contributions}

Conceptualization: Rhee CK. Methodology: Rhee CK, Ban WH. Formal analysis: Rhee CK. Writing - original draft preparation: Rhee CK, Ban WH. Writing - review and editing: Rhee CK, Ban WH. Approval of final manuscript: all authors.

\section{Conflicts of Interest}

CK Rhee received consulting/lecture fees from MSD, AstraZeneca, GSK, Novartis, Takeda, Mundipharma, Boehringer-Ingelheim, Teva, Sanofi, and Bayer. CK Rhee serves as deputy editor of the Tuberculosis of Respiratory Diseases, but has no role in the decision to publish this article. All remaining authors have declared no conflicts of interest.

\section{Funding}

No funding to declare.

\section{References}

1. Vogelmeier CF, Criner GJ, Martinez FJ, Anzueto A, Barnes PJ, Bourbeau J, et al. Global strategy for the diagnosis, management, and prevention of chronic obstructive lung disease 2017 report. GOLD executive summary. Am J Respir Crit Care Med 2017; 195:557-82.

2. Lozano R, Naghavi M, Foreman K, Lim S, Shibuya K,
Aboyans $\mathrm{V}$, et al. Global and regional mortality from 235 causes of death for 20 age groups in 1990 and 2010: a systematic analysis for the Global Burden of Disease Study 2010. Lancet 2012;380:2095-128.

3. Fabbri LM, Luppi F, Beghe B, Rabe KF. Complex chronic comorbidities of COPD. Eur Respir J 2008;31:204-12.

4. Barnes PJ, Celli BR. Systemic manifestations and comorbidities of COPD. Eur Respir J 2009;33:1165-85.

5. Barnes PJ. Inflammatory mechanisms in patients with chronic obstructive pulmonary disease. J Allergy Clin Immunol 2016;138:16-27.

6. Lushchak VI. Free radicals, reactive oxygen species, oxidative stress and its classification. Chem Biol Interact 2014;224:164-75.

7. Finkel T. Signal transduction by reactive oxygen species. J Cell Biol 2011;194:7-15.

8. Circu ML, Aw TY. Reactive oxygen species, cellular redox systems, and apoptosis. Free Radic Biol Med 2010;48:749-62.

9. Moodie FM, Marwick JA, Anderson CS, Szulakowski P, Biswas SK, Bauter MR, et al. Oxidative stress and cigarette smoke alter chromatin remodeling but differentially regulate NF-kappaB activation and proinflammatory cytokine release in alveolar epithelial cells. FASEB $J$ 2004;18:1897-9.

10. Ma Q. Role of nrf2 in oxidative stress and toxicity. Annu Rev Pharmacol Toxicol 2013;53:401-26.

11. Boutten A, Goven D, Artaud-Macari E, Boczkowski J, Bonay M. NRF2 targeting: a promising therapeutic strategy in chronic obstructive pulmonary disease. Trends Mol Med 2011;17:363-71.

12. Rangasamy $T$, Misra V, Zhen L, Tankersley CG, Tuder RM, Biswal S. Cigarette smoke-induced emphysema in $A / J$ mice is associated with pulmonary oxidative stress, apoptosis of lung cells, and global alterations in gene expression. Am J Physiol Lung Cell Mol Physiol 2009;296:L888-900.

13. Bosken $\mathrm{CH}$, Wiggs $\mathrm{BR}$, Pare $\mathrm{PD}$, Hogg JC. Small airway 
dimensions in smokers with obstruction to airflow. Am Rev Respir Dis 1990;142:563-70.

14. Hogg JC, Timens W. The pathology of chronic obstructive pulmonary disease. Annu Rev Pathol 2009;4:435-59.

15. lizuka $T$, Ishii $Y$, Itoh $K$, Kiwamoto $T$, Kimura $T$, Matsuno $Y$, et al. Nrf2-deficient mice are highly susceptible to cigarette smoke-induced emphysema. Genes Cells 2005;10:1113-25.

16. Ishii $Y$, Itoh $K$, Morishima $Y$, Kimura $T$, Kiwamoto $T$, lizuka $\mathrm{T}$, et al. Transcription factor Nrf2 plays a pivotal role in protection against elastase-induced pulmonary inflammation and emphysema. J Immunol 2005;175:6968-75.

17. Sussan TE, Rangasamy T, Blake DJ, Malhotra D, El-Haddad H, Bedja D, et al. Targeting Nrf2 with the triterpenoid CDDO-imidazolide attenuates cigarette smoke-induced emphysema and cardiac dysfunction in mice. Proc Natl Acad Sci U S A 2009;106:250-5.

18. Goven D, Boutten A, Lecon-Malas V, Marchal-Somme J, Amara N, Crestani B, et al. Altered Nrf2/Keap1-Bach1 equilibrium in pulmonary emphysema. Thorax 2008;63: 916-24.

19. Suzuki $M$, Betsuyaku $T$, Ito $Y$, Nagai $K$, Nasuhara $Y$, Kaga $K$, et al. Down-regulated NF-E2-related factor 2 in pulmonary macrophages of aged smokers and patients with chronic obstructive pulmonary disease. Am J Respir Cell Mol Biol 2008;39:673-82.

20. Donaldson GC, Seemungal TA, Bhowmik A, Wedzicha JA. Relationship between exacerbation frequency and lung function decline in chronic obstructive pulmonary disease. Thorax 2002;57:847-52.

21. Suissa S, Dell'Aniello S, Ernst P. Long-term natural history of chronic obstructive pulmonary disease: severe exacerbations and mortality. Thorax 2012;67:957-63.

22. Wedzicha JA, Seemungal TA. COPD exacerbations: defining their cause and prevention. Lancet 2007;370:78696.

23. Berenson CS, Kruzel RL, Eberhardt E, Sethi S. Phagocytic dysfunction of human alveolar macrophages and severity of chronic obstructive pulmonary disease. J Infect Dis 2013;208:2036-45.

24. Harvey CJ, Thimmulappa RK, Sethi S, Kong X, Yarmus $\mathrm{L}$, Brown $\mathrm{RH}$, et al. Targeting Nrf2 signaling improves bacterial clearance by alveolar macrophages in patients with COPD and in a mouse model. Sci Transl Med 2011;3:78ra32.

25. Cho HY, Imani F, Miller-DeGraff L, Walters D, Melendi GA, Yamamoto M, et al. Antiviral activity of Nrf2 in a murine model of respiratory syncytial virus disease. Am J Respir Crit Care Med 2009;179:138-50.

26. Foronjy RF, Dabo AJ, Taggart CC, Weldon S, Geraghty P. Respiratory syncytial virus infections enhance cigarette smoke induced COPD in mice. PLoS One 2014;9:e90567.

27. Wise RA, Holbrook JT, Criner G, Sethi S, Rayapudi S, Sudini KR, et al. Lack of effect of oral sulforaphane administration on Nrf2 expression in COPD: a randomized, double-blind, placebo controlled trial. PLoS One 2016;11: e0163716.

28. Marcoa R, Rodrigues DM, Dias M, Ladeira I, Vaz AP, Lima $R$, et al. Classification of chronic obstructive pulmonary disease (COPD) according to the new Global Initiative for Chronic Obstructive Lung Disease (GOLD) 2017: Comparison with GOLD 2011. COPD 2018;15:21-6.

29. Min T, Bodas M, Mazur S, Vij N. Critical role of proteostasis-imbalance in pathogenesis of COPD and severe emphysema. J Mol Med (Berl) 2011;89:577-93.

30. Mirza S, Clay RD, Koslow MA, Scanlon PD. COPD guidelines: a review of the 2018 GOLD report. Mayo Clin Proc 2018;93:1488-502.

31. Sun XJ, Li ZH, Zhang Y, Zhou G, Zhang JQ, Deng JM, et al. Combination of erythromycin and dexamethasone improves corticosteroid sensitivity induced by CSE through inhibiting PI3K-delta/Akt pathway and increasing GR expression. Am J Physiol Lung Cell Mol Physiol 2015;309:L139-46.

32. An TJ, Rhee CK, Kim JH, Lee YR, Chon JY, Park CK, et al. Effects of macrolide and corticosteroid in neutrophilic asthma mouse model. Tuberc Respir Dis 2018;81:80-7.

33. Yamamoto $T$, Yoh K, Kobayashi A, Ishii Y, Kure S, Koyama $A$, et al. Identification of polymorphisms in the promoter region of the human NRF2 gene. Biochem Biophys Res Commun 2004;321:72-9.

34. Masuko H, Sakamoto $T$, Kaneko $Y$, lijima H, Naito $T$, Noguchi E, et al. An interaction between Nrf2 polymorphisms and smoking status affects annual decline in FEV1: a longitudinal retrospective cohort study. BMC Med Genet 2011;12:97.

35. Yamada K, Asai K, Nagayasu F, Sato K, ljiri N, Yoshii N, et al. Impaired nuclear factor erythroid 2-related factor 2 expression increases apoptosis of airway epithelial cells in patients with chronic obstructive pulmonary disease due to cigarette smoking. BMC Pulm Med 2016;16:27.

36. Fratta Pasini AM, Ferrari M, Stranieri C, Vallerio P, Mozzini C, Garbin U, et al. Nrf2 expression is increased in peripheral blood mononuclear cells derived from mild-moderate ex-smoker COPD patients with persistent oxidative stress. Int J Chron Obstruct Pulmon Dis 2016;11:173343.

37. Ban WH, Kang HH, Kim IK, Ha JH, Joo H, Lee JM, et al. Clinical significance of nuclear factor erythroid 2-related factor 2 in patients with chronic obstructive pulmonary disease. Korean J Intern Med 2018;33:745-52. 